\title{
Oclusão da Comunicação Interatrial com a Nova Prótese Atriasept-CARDIA: Experiência Inicial
}

\author{
Francisco Chamiée $1-3$, Daniel Chamié4, Sergio Ramos ${ }^{2,3}$, Luiz Carlos Simões ${ }^{5}$, Raul Ivo Rossi Filho ${ }^{6}$ \\ João Carlos Tress ${ }^{7}$, Maximiliano Lacoste ${ }^{8}$, Fernanda Estefan Vivas ${ }^{9}$
}

\section{RESUMO}

Introdução: Neste trabalho, é apresentada a experiência inicial do grupo com a prótese Atriasept-CARDIA ${ }^{\text {TM }}$ para o fechamento de comunicação interatrial (CIA). É descrita a técnica de implante e são analisadas as características desse dispositivo. Método: Escolhemos pacientes que apresentavam defeitos com diâmetro estático não maior que $15 \mathrm{~mm}$, sem hipertensão arterial pulmonar grave ou outros defeitos congênitos passíveis de correção cirúrgica. Resultados: De maio de 2007 a dezembro de 2008, oito pacientes preencheram os critérios de inclusão. O implante foi possível em todos os casos. O diâmetro dos dispositivos implantados foi de $12 \mathrm{~mm}$ em dois casos, de $14 \mathrm{~mm}$ em um, de $20 \mathrm{~mm}$ em dois, e de $22 \mathrm{~mm}$ em três. Não houve qualquer complicação digna de nota e nenhum óbito. Um paciente apresentou pequeno shunt residual imediatamente após o procedimento, que desapareceu ao ecocardiograma transesofágico de controle aos seis meses. Conclusões: O fechamento de CIA com a prótese Atriasept-CARDIA ${ }^{\mathrm{TM}}$ demonstrou ser seguro e eficaz, apesar de ser tecnicamente mais complexo. Todas as modificações implementadas foram benéficas, especialmente as realizadas no dispositivo de quarta geração (daisy device). Seu uso ficou mais fácil e menos traumático para as estruturas cardíacas. Apesar dos resultados encorajadores obtidos, considerando-se a pequena série

\section{ABSTRACT} Percutaneous Closure of Atrial Septal Defect with
New Atriasept-CARDIA Device: Early Experience

Background: The authors's early experience with AtriaseptCARDIA $^{\text {TM }}$ for the closure of atrial septal defects (ASD) is reported. Procedural implant technique is described and some technical aspects of this new device are analyzed. Methods: Selection criteria included defects with static diameter up to $15 \mathrm{~mm}$ on transesophageal echocardiography, without significant pulmonary hypertension, in absence of other congenital cardiac defects requiring surgical therapy. Results: From May 2007 to December 2008, eight patients fulfilled the inclusion criteria and were submitted to percutaneous closure. Catheter closure was possible in all cases. Device diameters were $12 \mathrm{~mm}$ in two cases, $14 \mathrm{~mm}$ in one, $20 \mathrm{~mm}$ in two and $22 \mathrm{~mm}$ in three. Two second generation devices were utilized in two cases, third generation devices in five and fourth generation device in one. Follow-up time varied from 2 to 20 months (12.6 \pm 6.6 months). There were no deaths or major complications. One patient presented a small residual shunt immediately after procedure that was completely closed at the 6-month follow-up transesophageal echocardiography. Conclusions: ASD closure with the AtriaseptCARDIA $^{\text {TM }}$ ASD device showed to be safe and effective,

\footnotetext{
1 Setor de Hemodinâmica Pediátrica do Hospital dos Servidores do Estado, MS-RJ - Rio de Janeiro, RJ, Brasil.

${ }^{2}$ CARPE - Cardiologia Pediátrica e Fetal - Rio de Janeiro, RJ, Brasil.

3 INTERCAT - Cardiologia Intervencionista - Rio de Janeiro, RJ, Brasil.

4 Instituto Dante Pazzanese de Cardiologia - São Paulo, SP, Brasil.

${ }^{5}$ Instituto Nacional de Cardiologia de Laranjeiras - Rio de Janeiro, RJ,

Brasil.

${ }^{6}$ Instituto de Cardiologia de Porto Alegre - Porto Alegre, RS, Brasil.

7 Hospital de Clínicas de Niterói - Niterói, RJ, Brasil.

${ }^{8}$ Hospital Quinta D'Or - Rio de Janeiro, RJ, Brasil.

${ }^{9}$ Hospital dos Servidores do Estado MS - Rio de Janeiro, RJ, Brasil. Correspondência: Francisco José Araújo Chamié Queiroz. Av. Borges de Medeiros, 3501/103 - Lagoa - Rio de Janeiro, RJ, Brasil CEP 22470-001

E-mail: fchamie@pobox.com

Recebido em: 3/1/2009 • Aceito em: 12/3/2009
} 
de casos e a variabilidade dos dispositivos utilizados, mais estudos são necessários para estabelecer a segurança e a efetividade deste novo dispositivo, que parece ser uma excelente alternativa às próteses de malha de nitinol.

DESCRITORES: Defeitos do septo interatrial. Cateterismo cardíaco. Próteses e implantes. Ecocardiografia.

D sde os trabalhos pioneiros de King et al. ${ }^{1}$, em 1974, tem sido buscada uma alternativa nãocirúrgica confiável para o fechamento dos defeitos cardíacos.

Inúmeras próteses foram utilizadas para esse fim, sendo as mais usadas ainda hoje as próteses CardioSeal ${ }^{\mathrm{TM}} / \mathrm{Starflex}^{\mathrm{TM}}$ (NMT Medical Inc., Boston, Estados Unidos)2 ${ }^{2}$, Helex Septal Occluder ${ }^{\mathrm{TM}}$ (W.L. Gore \& Associates, Newark, Estados Unidos) ${ }^{3}$ e Amplatzer Septal Occluder ${ }^{\top M}$ (AGA Medical Corp., Golden Valley, Estados Unidos) ${ }^{4-6}$.

Apesar dos excelentes resultados obtidos a médio e longo prazos, algumas complicações, principalmente no fechamento da comunicação interatrial tipo ostium secundum, ainda tornam desejável a busca por outro dispositivo, que, agregando valores, não apresente algumas das características negativas dos dispositivos anteriores $^{7-9}$.

A prótese Atriasept-CARDIA ${ }^{\text {TM }}$ foi exaustivamente testada nos fechamentos de forame oval patente ${ }^{10,11}$, lançando as bases para seu aproveitamento na oclusão das comunicações interatriais.

O objetivo deste trabalho é apresentar a experiência inicial dos autores com o uso da prótese Atriasept-CARDIA ${ }^{\text {TM }}$ no fechamento percutâneo das comunicações interatriais únicas de tamanho pequeno a moderado.

\section{MÉTODO}

\section{Desenho do estudo}

Este estudo apresenta uma análise prospectiva, de único centro, dos pacientes portadores de comunicação interatrial submetidos a fechamento percutâneo com prótese Atriasept-CARDIA ${ }^{\mathrm{TM}}$, no período de maio de 2007 a janeiro de 2008. São descritas as características do novo dispositivo com as modificações sofridas até sua versão final, a técnica do implante e seus resultados.

\section{Seleção de pacientes}

No período mencionado, os pacientes portadores de comunicação interatrial tipo ostium secundum com although more technically demanding. Modifications implemented in the device were all beneficial, especially the ones in the fourth generation rounded device (daisydevice). They made the device easy to charge and less traumatic to cardiac structures. Despite the encouraging results obtained, considering the small number of cases, and the diversity of devices used, further studies are required to accurately establish safety and effectiveness of this new device that seems to be an excellent alternative to the nitinol cage larger devices.

DESCRIPTORS: Heart septal defects, atrial. Heart catheterization. Prostheses and implants. Echocardiography.

repercussão hemodinâmica e sem hipertensão pulmonar fixa, acima dos seis anos de vida, com peso mínimo de $15 \mathrm{~kg}$ e com diâmetro estático do defeito septal igual ou inferior a $15 \mathrm{~mm}$ foram, consecutivamente, submetidos a fechamento percutâneo com prótese Atriasept-CARDIA ${ }^{\text {TM }}$ em nosso Serviço. O diagnóstico dos defeitos foi feito por meio de ecocardiogramas transtorácicos e foram realizados ecocardiogramas transesofágicos para análise morfológica do defeito, identificação das bordas e medição definitiva do diâmetro da estenose para indicar a intervenção.

\section{A evolução da prótese Atriasept-CARDIA ${ }^{\mathrm{TM}}$}

Os dispositivos de oclusão utilizados neste trabaIho foram as próteses Atriasept-CARDIA ${ }^{\text {TM }}$ para comunicação interatrial (Cardia Inc., Eagan, Estados Unidos), nas três versões disponíveis em nosso meio (Figura 1).

Esse dispositivo é uma prótese de duplo disco, de baixo perfil, com mecanismo autocentrável, totalmente reposicionável e retirável, já na sua quarta versão ${ }^{12-14}$.

A primeira versão era composta de dois discos, quadrados, de polivinil álcool (Ivalon ${ }^{T M}$ ) de $2 \mathrm{~mm}$ de espessura, sustentados por quatro hastes de nitinol com reforços de titânio nas extremidades. Essa versão não esteve disponível no Brasil.

$\mathrm{Na}$ segunda versão, as quatro hastes foram reforçadas por metal trançado (nitinol), e o disco esquerdo foi montado pelo lado de fora da parte metálica, para reduzir o risco de trombose. Houve acréscimo de duas hastes por disco, criando uma forma hexagonal, com as hastes formando a bissetriz dos ângulos do poliedro. O revestimento dos discos ainda era composto de Ivalon $^{\mathrm{TM}}$, embora mais fino que na versão anterior. O mecanismo centralizador era composto de fios de nitinol, conectando a extremidade das hastes dos dois discos (Figura 2).

A terceira versão, ainda hexagonal, trouxe pequenas modificações com relação à primeira: no disco esquerdo foi introduzido um anel de nitinol, conectando as extremidades de todas as hastes e fazendo com que elas se comportassem como uma peça única, mini- 


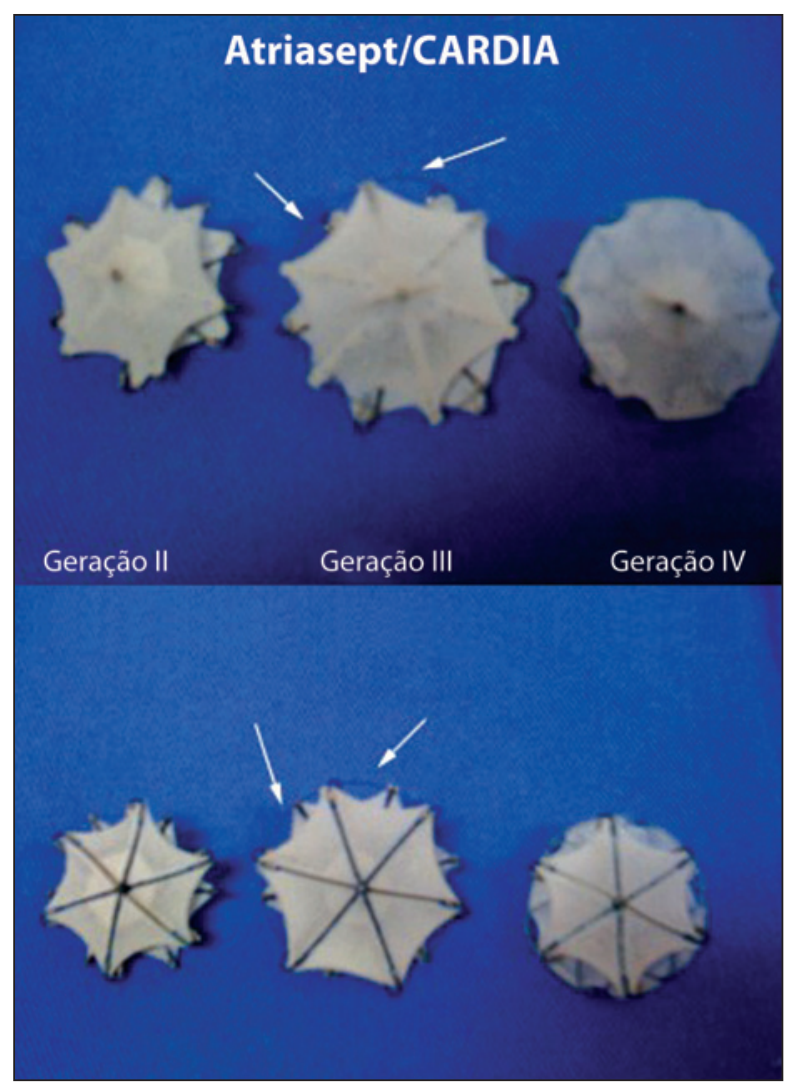

Figura 1 - Alinhados, da esquerda para a direita, os modelos de prótese CARDIA II, CARDIA III e CARDIA IV. No painel superior, a visão do disco esquerdo. No painel inferior, a visão do disco direito.

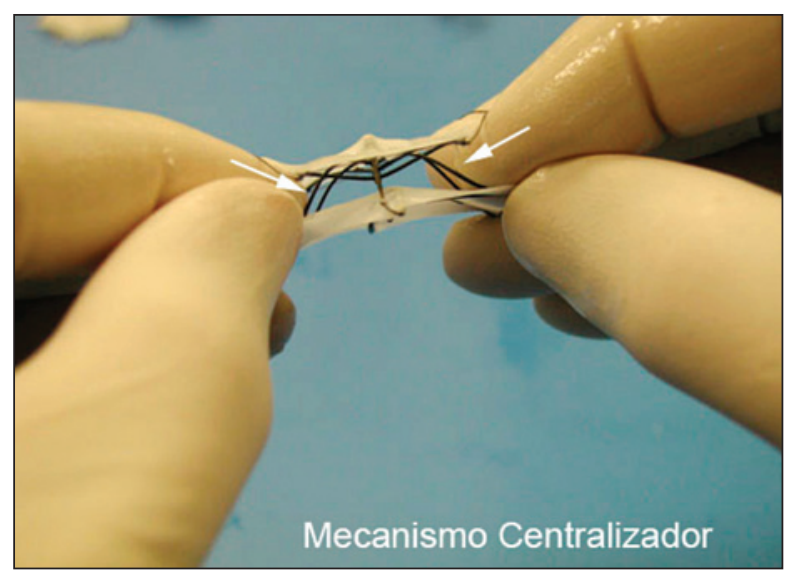

Figura 2 - Detalhes do mecanismo de centralização. Observam-se os fios de nitinol conectando os discos direito e esquerdo.

mizando a possibilidade de prolapso inadvertido de uma haste através do defeito, o que acontecia, por vezes, no primeiro modelo. O mecanismo centralizador era idêntico ao anterior (Figura 3).

A quarta versão mostrou uma prótese completamente redonda do lado esquerdo. As hastes do disco

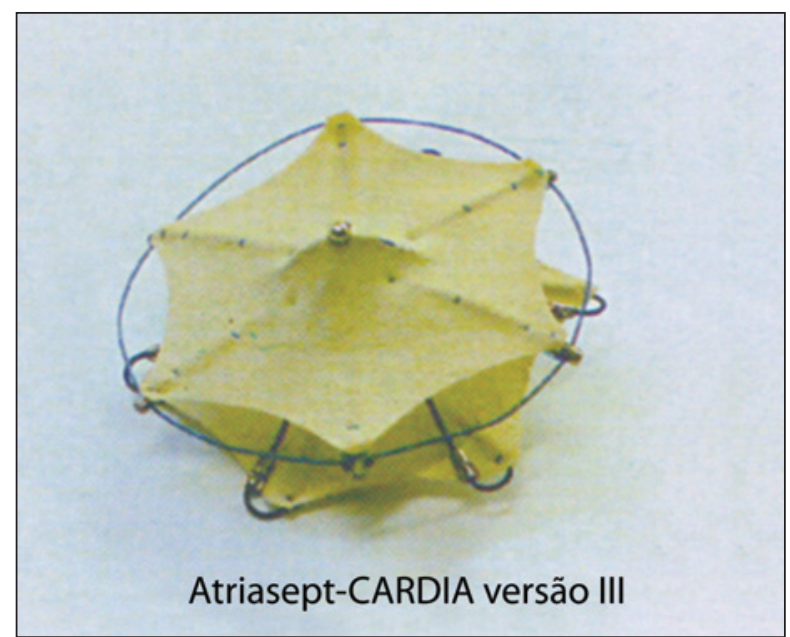

Figura 3 - Aproximação da versão III da prótese. Observe-se o metal das hastes por dentro da vela de Ivalon ${ }^{\mathrm{TM}}$. Em detalhe, também, o anel que conecta as hastes do disco esquerdo.

esquerdo ficaram duplas, dobradas sobre si mesmas, formando uma alça. Dessa forma, ficaram menos traumáticas, sem pontas agudas, dando ao disco o aspecto de uma margarida (daisy device). O disco direito e o mecanismo centralizador permaneceram idênticos aos das versões anteriores.

As próteses atuais estão disponíveis de $10 \mathrm{~mm}$ a $34 \mathrm{~mm}$, em incrementos de $2 \mathrm{~mm}$, tamanhos que se referem ao diâmetro central da prótese. São colocadas com o auxílio de bainhas longas, de calibre de acordo com o dispositivo a ser implantado, como se segue: bainha $9 \mathrm{Fr}$ para prótese de $10 \mathrm{~mm}$; bainha $10 \mathrm{Fr}$ para próteses de $12 \mathrm{~mm}$ e $14 \mathrm{~mm}$; bainha $11 \mathrm{Fr}$ para próteses de $16 \mathrm{~mm}$ a $20 \mathrm{~mm}$; bainha $12 \mathrm{Fr}$ para próteses de $22 \mathrm{~mm}$ a $26 \mathrm{~mm}$; bainha $13 \mathrm{Fr}$ para próteses de $28 \mathrm{~mm}$ a $32 \mathrm{~mm}$; e, finalmente, bainha $14 \mathrm{Fr}$ para prótese de $34 \mathrm{~mm}$.

\section{Ecocardiograma transesofágico}

O defeito foi analisado da forma rotineira, visualizando-se as bordas aórtica, da veia cava superior, e inferior.

Além da ótima visualização do defeito e da mensuração de seu diâmetro estático, foi realizada rotineiramente análise dos fluxos e de toda a extensão do septo atrial, visando à detecção de mais de um orifício ou a presença de aneurisma.

\section{Técnica do procedimento}

Ácido acetilsalicílico (AAS), na dose de $5 \mathrm{mg} / \mathrm{kg}$ ou $100 \mathrm{mg}$ em adultos, era iniciado pelo menos 24 horas antes do procedimento.

Todos os implantes foram realizados sob anestesia geral, com intubação orotraqueal, e monitorados por ecocardiograma transesofágico. 
O acesso vascular foi obtido por meio de punção venosa femoral.

Os pacientes receberam heparinização sistêmica na dose de $100 \mathrm{UI} / \mathrm{kg}$ ou $5.000 \mathrm{UI}$ em adultos. Caso o procedimento durasse mais de uma hora, era administrada metade da dose original, a cada 30 minutos, até o término do exame.

Profilaxia antimicrobiana com cefalotina venosa era administrada na dose de $50 \mathrm{mg} / \mathrm{kg}$ (máximo de $2 \mathrm{~g}$ ) no momento do procedimento, seguida por mais duas doses de $25 \mathrm{mg} / \mathrm{kg}$ após.

Cateterismo das cavidades cardíacas direita e esquerda foi realizado de rotina, com registro das pressões em artéria pulmonar e átrios direito e esquerdo. Não foram realizados cálculos de fluxo durante os procedimentos de oclusão. O defeito septal foi cruzado com cateter diagnóstico (MPA2, 5 ou 6 Fr), posicionando guia rígido $0,035 " / 260 \mathrm{~cm}$ na veia pulmonar superior esquerda. Em seguida, foi introduzido balão medidor AGA (AGA Medical Corporation, Golden Valley, Estados Unidos) de $24 \mathrm{~mm}$ ou $34 \mathrm{~mm}$, conforme a medida do diâmetro estático do defeito. O balão foi insuflado até a completa oclusão do defeito (stopflow technique), realizando a medida de seu diâmetro estirado. Os resultados obtidos pelo ecocardiograma transesofágico e pela fluoroscopia foram comparados, sendo escolhido o mais adequado.

Os dispositivos são escolhidos pelo seu diâmetro central, que deve ser igual ou no máximo $1 \mathrm{~mm}$ a $2 \mathrm{~mm}$ maior que o diâmetro estirado do orifício. Nas próteses de até $22 \mathrm{~mm}$, o comprimento total das hastes é $14 \mathrm{~mm}$ maior.

Retirado o balão medidor, era introduzida sobre a guia uma bainha longa de Mullins de diâmetro apropriado para a prótese escolhida (12 Fr, 13 Fr ou $14 \mathrm{Fr}$ ), posicionada no interior da veia pulmonar. Tomava-se o cuidado de retirar todo o ar da bainha, abrindo-se a torneira lateral e retirando-se lentamente o dilatador, para permitir o sangramento espontâneo. Além disso, era introduzido, através da válvula hemostática, um dilatador curto de $10 \mathrm{Fr}$ (existente na embalagem da bainha), com uma seringa parcialmente cheia de solução salina em sua extremidade proximal, sendo feita nova aspiração de ar, com o especial cuidado de explorar a região entre a válvula hemostática e a junção com o corpo da bainha.

A prótese escolhida era imersa em solução salina durante alguns minutos.

Além da prótese, são fornecidos, também, um pequeno carregador de plástico transparente e um biótomo especial, com uma rosca que possibilita seu travamento na posição fechada.

A extremidade distal do biótomo era introduzida em um introdutor curto de calibre idêntico ao da bainha de Mullins e, sequencialmente, no carregador de plástico. A "garra" do biótomo era posicionada de forma a capturar o pino do disco direito da prótese, permitindo que a mesma pudesse girar livremente sobre seu eixo central, mesmo com o biótomo fechado. Em seguida, o biótomo era recuado cuidadosamente, recoIhendo os discos no carregador de plástico. Depois de posicionada totalmente dentro do carregador, a prótese era transferida para o introdutor curto e o carregador de plástico era descartado. A seguir, era realizada a lavagem do introdutor de forma a expulsar o ar contido na membrana dos discos.

Uma vez dentro do introdutor curto, a prótese estava pronta para ser transferida para a bainha de Mullins. Essa etapa era feita conectando-se a extremidade distal do introdutor curto através da válvula hemostática da bainha de Mullins. Após a passagem pela válvula, a prótese trafegava sem nenhuma resistência pela bainha longa e era posicionada na sua extremidade distal. Nesse ponto, a bainha de Mullins era recuada até o corpo do átrio esquerdo e, nesse local, exteriorizado o disco esquerdo da prótese. Em seguida, era aberta a porção central e, sob visão ecocardiográfica, encaixada no defeito septal, colocando o disco esquerdo em aposição à superfície esquerda do septo atrial. O dispositivo era mantido cuidadosamente nessa posição e a bainha de Mullins era recuada ainda mais, expondo totalmente o disco direito. Nesse momento, o cabo do biótomo era empurrado de encontro ao septo, configurando completamente o disco direito e fechando a prótese em posição.

Se a fluoroscopia e o ecocardiograma transesofágico mostrassem boa estabilidade do dispositivo e ausência de shunt residual, a prótese era liberada, desenroscando a trava do biótomo e abrindo suas garras (Figura 4). É importante ressaltar que, após a abertura das garras, o biótomo era recuado mantendo a posição aberta até ser introduzido no interior da bainha de Mullins, para prevenir dano inadvertido a cordoalhas ou folhetos da valva tricúspide ou mesmo a outras estruturas do átrio direito.

\section{Seguimento}

O acompanhamento era realizado por meio de ecocardiograma transtorácico no dia seguinte ao procedimento, antes da alta hospitalar, e com 1, 3 e 12 meses. Em adição, ecocardiograma transesofágico era realizado no sexto mês.

AAS $5 \mathrm{mg} / \mathrm{kg} /$ dia ou $100 \mathrm{mg} / \mathrm{dia}$, nos adultos, era mantido até o ecocardiograma transesofágico no sexto mês, caso o defeito estivesse completamente fechado.

Profilaxia antimicrobiana para endocardite infecciosa era mantida por seis meses. Caso persistisse shunt residual, a profilaxia deveria ser mantida até seu desaparecimento ou pelo resto da vida. 

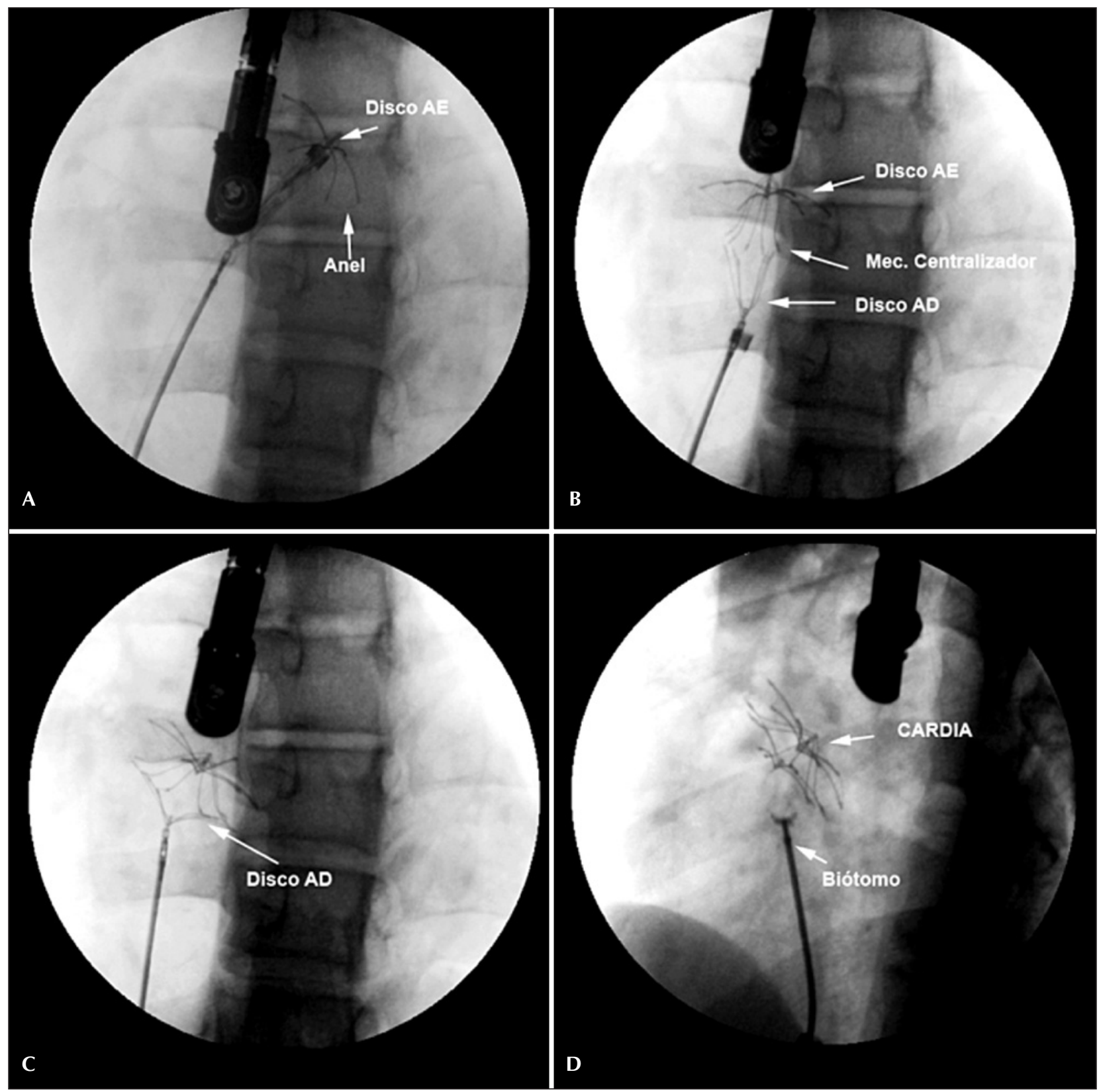

Figura 4 - Imagens angiográficas do implante. A: Disco esquerdo imediatamente após sua exteriorização no corpo do átrio esquerdo. Notese o anel conectando as hastes, na versão III do dispositivo. B: Disco esquerdo em aposição ao septo atrial; a porção central está exposta no interior do defeito. C: Empurrando-se o biótomo, o disco direito é configurado. D: Após certificar-se de que a prótese está bem posicionada, o biótomo é aberto, liberando completamente a prótese.

\section{Análise estatística}

Na análise estatística descritiva, as variáveis contínuas são expressas como média e desvio padrão e as variáveis categóricas, como números e porcentagens.

\section{RESULTADOS}

No período de maio de 2007 a dezembro de 2008, 8 pacientes preencheram os critérios de inclusão e foram submetidos ao procedimento. Eram todos clini- camente assintomáticos, à exceção de um paciente de 25 anos (paciente 6 - WLC), do sexo masculino, que apresentava hemiparesia do dimídio direito como sequela de acidente vascular cerebral isquêmico prévio.

Do total de pacientes, 5 eram do sexo masculino e 3, do sexo feminino. As idades variaram de 10 a 50 anos $(24,7 \pm 17,3$ anos $)$.

A borda aórtica estava presente em 3 pacientes e ausente nos outros 5. Nenhum paciente apresen- 
tou septo atrial aneurismático ou múltiplas fenestrações.

Os diâmetros estáticos obtidos ao ecocardiograma transesofágico, variaram de $8 \mathrm{~mm}$ a $15 \mathrm{~mm}(12 \pm$ 2,4 mm) e os diâmetros estirados variaram de $11 \mathrm{~mm}$ a $21 \mathrm{~mm}(16,2 \pm 4,1 \mathrm{~mm})$.

O implante foi possível em todos os casos, sendo utilizadas 9 próteses nos 8 pacientes. Durante o carregamento no introdutor curto, uma prótese de $22 \mathrm{~mm}$ teve seu disco esquerdo danificado, sendo substituída por uma de $20 \mathrm{~mm}$ (na ausência de outra do mesmo diâmetro), que foi implantada sem anormalidades e com bom resultado.

O diâmetro dos dispositivos implantados foi de $12 \mathrm{~mm}$ em dois casos, $14 \mathrm{~mm}$ em um caso, $20 \mathrm{~mm}$ em dois, e $22 \mathrm{~mm}$ em três (Tabela 1).

Foram empregadas próteses da versão II em dois casos, da versão III em cinco, e da versão IV em um.

Não houve qualquer complicação significativa e nenhum óbito.

\section{Seguimento}

O tempo de seguimento variou de 2 a 20 meses $(16,6 \pm 6,6$ meses). Não houve ocorrência de óbito. Arritmias e distúrbios de condução também não foram observados em nenhum momento do seguimento.

No seguimento ecocardiográfico, não se observou presença de trombos, vegetações, deslocamento ou embolização da prótese. Interferência com outras estruturas cardíacas na proximidade do septo interatrial também não foi identificada.

Um paciente apresentou pequeno shunt residual, imediatamente após o procedimento. No seguimento de um ano, notou-se oclusão completa e espontânea do defeito.

\section{DISCUSSÃO}

A limitação das próteses de disco (Cardio-Seal ${ }^{\mathrm{TM}}$ / Starflex ${ }^{\mathrm{TM}}$ e Helex ${ }^{\mathrm{TM}}$ ), que não permitem o fechamento de defeitos maiores que 20 mm, em adição à grande quantidade de metal (com perfil relativamente alto) e o potencial risco (ainda que pequeno) de erosão ocorrendo nas próteses Amplatzer ${ }^{\mathrm{TM}}$ de grande tamanho ${ }^{15}$ tornam desejável a existência de uma alternativa para esses dispositivos.

Embora tenha sido utilizada em várias pequenas séries de casos, principalmente na Europa, existem poucos trabalhos publicados sobre o fechamento de comunicação interatrial com uso da prótese Atriasept-CARDIA ${ }^{\mathrm{TM}}$.

Essa prótese é um dispositivo de duplo disco, com baixo perfil e mecanismo de autocentralização, completamente capturável e reposicionável. Pode ser capaz de fechar defeitos maiores que os demais dispositivos de duplo disco. O modelo IV, correntemente comercializado, está disponível em tamanhos de $10 \mathrm{~mm}$ a $32 \mathrm{~mm}$. Há um caso relatado por Goy, de Lausanne, Suíça, em quem foi realizado o fechamento de um defeito de $38 \mathrm{~mm}$, com sucesso, utilizando um dispositivo construído sob encomenda (Denis Haagen, comunicação pessoal).

Até o momento, não há dados precisos sobre o número de implantes realizados com essa prótese, mas os fabricantes estimam que tenham sido realizados 480 fechamentos de comunicação interatrial com a versão III e pelo menos $115 \mathrm{com}$ a prótese redonda (versão IV). Foram relatadas algumas complicações nos implantes realizados na Europa e nos Estados Unidos: um dispositivo migrou após o implante e foi recuperado no mesmo procedimento. O operador avaliou que o dispositivo foi escolhido erroneamente, sendo pequeno demais para o defeito. Houve, também, 8 perfurações de aorta ou átrio com o dispositivo III (Denis Haagen, comunicação pessoal), sendo 2 no Brasil (Carlos Pedra, comunicação pessoal).

TABELA 1

Características demográficas e hemodinâmicas dos pacientes que receberam próteses $(\mathbf{n}=8)$

\begin{tabular}{|c|c|c|c|c|c|c|c|c|}
\hline № & Identificação & Sexo & $\begin{array}{l}\text { Idade } \\
\text { (anos) }\end{array}$ & $\begin{array}{c}\text { Peso } \\
(\mathrm{kg})\end{array}$ & $\begin{array}{c}\text { PAP } \\
(\mathrm{mmHg})\end{array}$ & $\begin{array}{l}\text { Diâmetro estático } \\
(\mathbf{m m})\end{array}$ & $\begin{array}{l}\text { Diâmetro estirado } \\
(\mathbf{m m})\end{array}$ & $\begin{array}{c}\text { Prótese } \\
\text { Versão/Diâmetro }(\mathrm{mm})\end{array}$ \\
\hline 1 & $\mathrm{BCM}$ & $\mathrm{F}$ & 12 & 40 & 25/11 (18) & 13 & 19 & $\mathrm{II} / 22$ \\
\hline 2 & MMS & M & 11 & 22 & $35 / 18(24)$ & 11 & 11 & $\mathrm{II} / 12$ \\
\hline 3 & ILMC & $M$ & 17 & 62 & ni & 13 & 19 & III/20 \\
\hline 4 & PCG & M & 22 & 70 & ni & 15 & 20 & III/22 \\
\hline 5 & RSLR & M & 10 & 31 & $26 / 15(20)$ & 9 & 10 & III/12 \\
\hline 6 & WLC & M & 25 & 70 & $31 / 14(23)$ & 14 & 20 & III/20 \\
\hline 7 & AJPS & $\mathrm{F}$ & 50 & 56 & ni & 13 & 21 & III/22 \\
\hline 8 & MKKS & $\mathrm{F}$ & 48 & 70 & $34 / 14(21)$ & 8 & 14 & IV/14 \\
\hline
\end{tabular}

$\mathrm{F}=$ feminino; $\mathrm{M}=$ masculino; $\mathrm{n}=$ número de pacientes; $\mathrm{ni}=$ não informado; $\mathrm{PAP}=$ pressão arterial pulmonar. 
Outras complicações relatadas foram derrame pericárdico pequeno, que não necessitou drenagem, em um caso, e regurgitação mitral leve, que desapareceu aos três meses de seguimento em outro ${ }^{14}$.

Embora a técnica de implante não seja mais difícil que a das próteses Amplatzer ${ }^{\mathrm{TM}}$ ou Helex ${ }^{\mathrm{TM}}$, exige um pouco mais de atenção e cuidado na colocação do disco esquerdo, que precisa "abraçar" a aorta, de forma que as hastes que ficam acima dela realmente deslizem livremente sobre a superfície aórtica. Caso elas fiquem "apontando" diretamente para a aorta, existe a possibilidade de perfuração com a formação de fístula aortoatrial, como já tivemos oportunidade de testemunhar em um caso que nos foi trazido para avaliação.

É importante ressaltar que todas as perfurações relatadas foram com a versão III, na qual as hastes ainda eram retas. As modificações introduzidas na versão IV a tornam muito segura e praticamente incapaz de causar erosões, que não foram relatadas até este momento.

Como nas outras próteses, a deficiência da borda aórtica nas comunicações interatriais maiores muitas vezes faz com que o dispositivo fique perpendicular ao defeito, caindo no átrio direito com muita facilidade. Essa é uma dificuldade comumente encontrada. Em todos os nossos casos, pudemos corrigir a trajetória do disco esquerdo com simples rotação horária e abertura cuidadosa do mecanismo centralizador antes de tocar o septo. Em um caso, tivemos que manobrar durante algum tempo até conseguir a ótima posição da prótese. Apesar disso, ela sempre respondeu às manobras feitas e pôde ser reintroduzida na bainha longa e reconfigurada, sem qualquer dificuldade ou dano aos discos.

O resultado final do implante foi extremamente satisfatório em todos os casos (Figura 5).
Como a amostra é pequena e todos os casos tinham defeitos septais únicos, a ocorrência de apenas um shunt residual pode estar subestimada. O perfil do dispositivo é realmente muito baixo e fica pouco visível ao ecocardiograma transtorácico de controle, podendo passar despercebida para operadores menos experientes.

Apesar das muitas qualidades da prótese AtriaseptCARDIA $^{\mathrm{TM}}$, um dos dados negativos é a necessidade de bainhas de Mullins de grande diâmetro (12 Fr, $13 \mathrm{Fr}$ ou 14 Fr) para sua introdução. Por outro lado, seu uso facilita o implante, por manter melhor a curvatura posterior nos defeitos maiores e por possibilitar a correta realização das manobras necessárias para o realinhamento da prótese.

Da mesma forma que a prótese Amplatzer ${ }^{\mathrm{TM}}$, a medida do diâmetro correto do defeito é de suma importância e esse dispositivo não deve ser dimensionado além do necessário. Caso isso ocorra, a prótese não se configurará corretamente e os discos ficarão algo "arqueados", não mantendo a aposição necessária ao septo interatrial. Nesse caso, o dispositivo deverá ser retirado e substituído por um de menor tamanho.

A prótese Atriasept-CARDIA ${ }^{\mathrm{TM}}$ é bastante resistente ao uso e pode ser reintroduzida na bainha e reconfigurada no septo tantas vezes quantas necessárias, sem qualquer problema. Entretanto, muita cautela deve ser exercida na passagem pela válvula hemostática durante sua retirada da bainha, mormente se há a intenção de reutilizá-la. Duas possibilidades podem ocorrer: quebra do pino de conexão onde se fixa a mandíbula do biótomo e perda de fixação das velas à moldura na passagem pela válvula hemostática. A retirada do dispositivo deve ser feita com extremo cuidado, e a lavagem e a inspeção criteriosas do material dos discos após a retirada devem ser enfatizadas antes da reutilização.

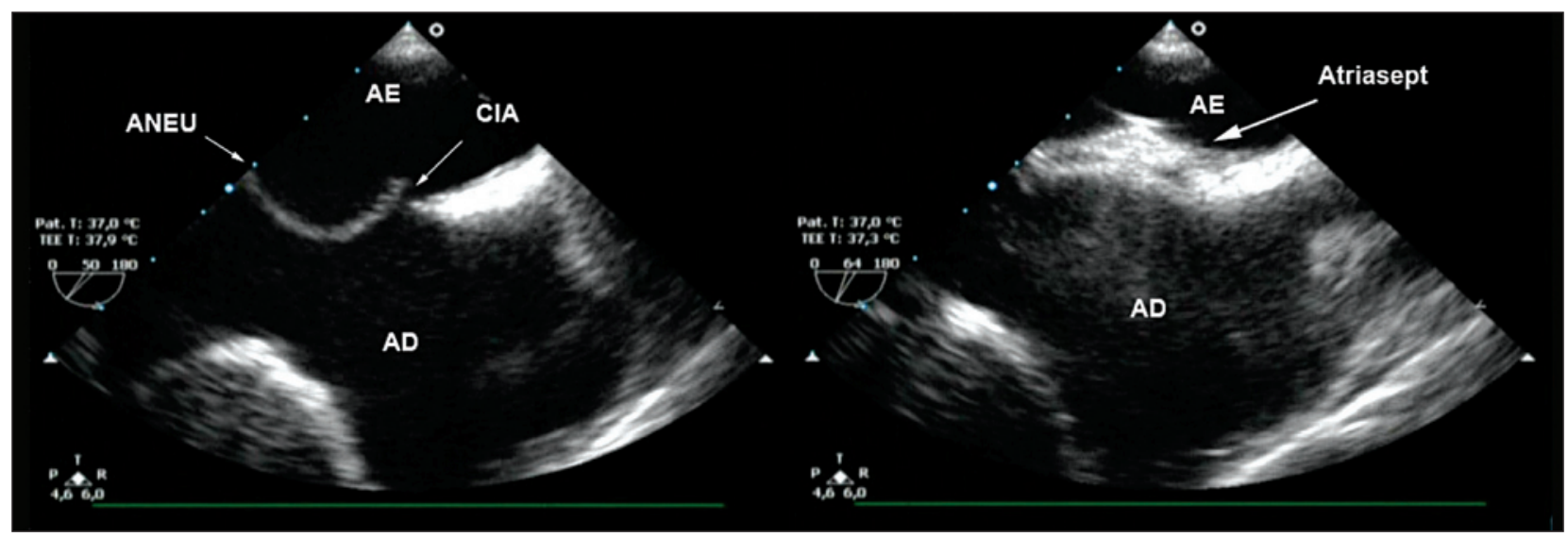

Figura 5 - Resultado final. À esquerda, a imagem da comunicação interatrial antes do fechamento. Observe-se a característica redundante do septo atrial sem, no entanto, configurar um aneurisma de septo atrial. À direita, o resultado final com a prótese corretamente implantada. Ressaltese o seu baixo perfil. $\mathrm{AD}=$ átrio direito; $\mathrm{AE}=$ átrio esquerdo; $\mathrm{ANEU}=$ aneurisma; $\mathrm{CI} A=$ comunicação interatrial. 


\section{CONCLUSÕES}

O fechamento de comunicações interatriais únicas, de tamanho pequeno a moderado, com a prótese Atriasept-CARDIA ${ }^{\mathrm{TM}}$ se mostrou seguro e eficaz, apesar de tecnicamente um pouco mais complexo que o observado com as próteses de malha de nitinol.

As modificações implementadas ao dispositivo foram todas positivas, especialmente às da quarta geração, que transformaram a prótese no modelo completamente arredondado, tornando o carregamento e o implante mais fáceis. As extremidades arredondadas diminuíram o risco de trauma às estruturas cardíacas.

Embora tenha o potencial de ocluir defeitos maiores com maior segurança que as próteses de duplo disco, as próteses Atriasept-CARDIA ${ }^{\mathrm{TM}}$ ainda não tiveram sua efetividade comprovada nos defeitos do septo atrial de grandes dimensões, em que as próteses Amplatzer ${ }^{\mathrm{TM}}$ já se mostraram eficazes e seguras.

Apesar dos resultados encorajadores obtidos, considerando-se a pequena série de casos e a variabilidade dos dispositivos utilizados, mais estudos são necessários para estabelecer a segurança e a efetividade desse dispositivo, bem como estabelecer os limites de indicação para seu uso.

\section{CONFLITO DE INTERESSES}

Francisco Chamié é consultor técnico e proctor para as firmas Bioassist (AGA) e Neomex (CARDIA). Essas atividades não apresentam conflito de interesses nem influenciaram os resultados apresentados. Os demais autores declararam inexistência de conflito de interesses.

\section{REFERÊNCIAS BIBLIOGRÁFICAS}

1. King TD, Thompson SL, Steiner C, Mills NL. Secundum atrial septal defect. Nonoperative closure during cardiac catheterization. JAMA. 1976;235(23):2506-9.

2. Carminati $M$, Chessa M, Butera G, Bini RM, Giusti S, Festa $P$, et al. Transcatheter closure of atrial septal defects with the Starflex device: early results and follow-up. J Interv Cardiol. $2001 ; 14(3): 319-24$.

3. Vincent RN, Raviele AA, Diehl HJ. Single-center experience with the Helex septal occluder for closure of atrial septal defects in children. J Interv Cardiol. 2003;16(1):79-82.

4. Chamié F, Chamié D, Ramos S, Tress JC, Victer R. Fechamento percutâneo das comunicações interatriais complexas. Rev Bras Cardiol Invas. 2006;14(1):47-55.

5. Chamié F, Chamié D, Ramos S, Tress JC, Victer R. Oclusão percutânea dos defeitos do septo atrial utilizando mais de uma prótese. Rev Bras Cardiol Invas. 2008;16(1):77-85.

6. Rossi RI, Cardoso CO, Machado PR, Francois LG, Horowitz ES, Sarmento-Leite R. Transcatheter closure of atrial septal defect with Amplatzer device in children aged less than 10 years old: immediate and late follow-up. Catheter Cardiovasc Interv. 2008;71(2):231-6.

7. Chessa M, Carminati M, Butera G, Bini RM, Drago M, Rosti $\mathrm{L}$, et al. Early and late complications associated with transcatheter occlusion of secundum atrial septal defect. J Am Coll Cardiol. 2002;39(6):1061-5.

8. Butera G, De Rosa G, Chessa M, Rosti L, Negura DG, Luciane $\mathrm{P}$, et al. Transcatheter closure of atrial septal defect in young children: results and follow up. J Am Coll Cardiol. 2003;42(2):241-5.

9. Lange SA, Schoen SP, Braun MU, Schulze MR, Boscheri AF, Kittner $\mathrm{T}$, et al. Perforation of aortic root as secondary complication after implantation of patent foramen ovale occlusion device in a 31-year-old woman. J Interv Cardiol. 2006;19(2):166-9.

10. Spies C, Strasheim R, Timmermanns I, Schraeder R. Patent foramen ovale closure in patients with cryptogenic thromboembolic events using the Cardia PFO occlude. Eur Heart J. $2006 ; 27(3): 365-71$.

11. Silva JMF, Pedra SRF, Fontes VF, Arnoni DG, Reyes RO, Pontes Jr. SC, et al. Experiência inicial com a utilização do dispositivo Cardia Intrasept ${ }^{\mathrm{TM}}$ no fechamento percutâneo do forame oval patente. Rev Bras Cardiol Invas. 2007;15(4):386-93.

12. Goy JJ, Stauffer JC, Yusoff Z, Wong AR, Owlya R, Perret F, et al. Percutaneous closure of atrial septal defect type ostium secundum using the new Intrasept occluder: initial experience. Catheter Cardiovasc Interv. 2006;67(2):265-7.

13. Chessa M, Butera G, Carminati M. Preliminary experiences closing secundum atrial septal defect using the modified Cardia Intrasept device. J Invasive Cardiol. 2007;19(3):142-4.

14. Turner D, Sabiniewicz R, Chessa M, Zurkurnai Y, Rahim A, Urena $\mathrm{P}$, et al. Initial experience with the Atriasept: a new device for transcatheter closure of secundum atrial septal defect. Cathet Cardiovasc Interv. 2007;70:8.

15. Amin Z, Hijazi ZM, Bass JL, Cheatham JP, Hellenbrandt WE, Kleinman CS. Erosion of Amplatzer septal occluder device after closure of secundum atrial septal defects: review of registry of complications and recommendations to minimize future risks. Catheter Cardiovasc Interv. 2004;63(4): 496-502. 